\title{
Determinants of response/non response to dietary fat interventions on biomarkers of insulin resistance
}

\author{
I. de Stanlaigh, AM. Murphy and Prof. H.M. Roche \\ Nutrigenomics Research Group, UCD Conway Institute for Biomolecular and Biomedical Sciences/UCD Institute of \\ Food and Health, School of Public Health, Physiotherapy and Sports Science, University College Dublin, Belfield, \\ Dublin 4
}

Variable responsiveness to dietary interventions is a significant challenge in terms of identifying effective therapies. Precision nutrition approaches attempt to define the right diet, for the right person at the right time. Dietary intervention studies investigating the effect of dietary fat intake on insulin sensitivity have demonstrated mixed results ${ }^{(1)}$. Typically $40 \%$ of cohorts respond ${ }^{(2)}$. Secondary analyses of these studies with large variability in cohort response to the dietary fat interventions provide the opportunity to understand potential determinants of responsiveness. While habitual fat intake, BMI and insulin resistance status have been highlighted as potential effectors of response ${ }^{(3-5)}$, additional research is required to determine the impact of metabolic-inflammatory phenotypes associated with effective interventions.

The LIPGENE study was a Pan European randomised dietary intervention study. Subjects with the metabolic syndrome were randomised to 4 diets for 12 weeks: high-SFA (HSFA), high-MUFA (HMUFA), low-fat high complex carbohydrate (LFHCC) and LFHCC with long chain (LC) n-3 PUFA(6). The aim of this post-hoc analysis was to identify determinants of response versus non-response with respect to insulin resistance.

The cohort $(\mathrm{n}=407)$ was split into 3 dietary response groups based on change in HOMA-IR post intervention: Non-responders (no change in HOMA-IR), responders with an increase in HOMA-IR (IS $\rightarrow$ IR) and responders with a decrease in HOMA-IR (IR $\rightarrow$ IS). The impact of a range of metabolic, inflammatory, anthropometric biomarkers in defining response was investigated.

Identical patterns of response to all diets indicated that factors other than the dietary intervention influenced response. Stepwise multiple regression analyses highlighted fasting insulin and C-peptide as strong predictors of a change in HOMA-IR (P<0.05). Higher pre-intervention C-peptide concentration predicted reduced HOMA-IR. Conversely, low C-peptide concentration predicted increased insulin resistance. Fasting insulin:C-peptide ratio was significantly different following intervention between responders who became more insulin sensitive (IR $\rightarrow$ IS) compared to both non-responders and responders who became more insulin resistant $(\mathrm{IS} \rightarrow \mathrm{IR})(\mathrm{P}=0.001)$. The ratio was lower in responders who became insulin more sensitive (IR $\rightarrow$ IS), compared to responders IS $\rightarrow$ IR who had increased fasting insulin:C-peptide ratio.

This analysis suggests that insulin:C-peptide ratio, reflective of pancreatic insulin secretion capacity, is an important predictor of responsiveness to dietary fat modification. These results are worthy of further investigation/validation given the key role of pancreatic functionality in the transition from insulin resistance to T2DM.

1. Tierney AC, Roche HM. Mol Nutr Food Res. 2007:51(10):1235-48.

2. Minihane AM, Vinoy S, Russell WR et al. Br J Nutr. 2015;114(07):999-1012

3. Lovejoy JC, Smith SR, Champagne CM et al. Diabetes Care. 2002;25(8):1283-8.

4. Yubero-Serrano EM, Delgado-Lista J, Tierney AC et al. Am J Clin Nutr. 2015;(6):1509-17.

5. Blanco-Rojo R, Alcala-Diaz JF, Wopereis S et al. Diabetologia. 2016;59(1):67-76.

6. Tierney a C, McMonagle J, Shaw DI et al. Int J Obes (Lond). 2011;35(6):800-9. 